\title{
An Electroactive Polymer Modified Separator with A Potential Clamp Function
}

\author{
Kaiyue Zhang ${ }^{1}$, Wei Xiao ${ }^{1, a}$, Jianguo Liu ${ }^{1}$ and Chuanwei Yan ${ }^{1}$ \\ ${ }^{1}$ Institute of Metal Research, Chinese Academy of Sciences, Shenyang 110016, China
}

\begin{abstract}
An inexpensive electroactive polymer polytriphenylamine (PTPAn) was synthesized through chemical oxidative polymerization of TPA. The composite separator was simply prepared by impregnating a commercial PP separator with PTPAn polymer solution. PTPAn was observed on the surface of the composite separator and formed a uniform coating. In cyclic voltammetry test, PTPAn polymer exhibited a pair of well-defined redox peaks between 3.0 and $4.5 \mathrm{~V}$, which could be regarded as a reversible electrochemical p-doping and dedoping process. When incorporated in a blocking cell, the composite separator was able to hold a constant potential for current densities up to $5 \mathrm{~mA} / \mathrm{cm}^{2}$ to protect the cell from overcharging.
\end{abstract}

\section{Introduction}

Overcharge is a condition in which charging current is applied to a battery that has reached its end-of-charge potential, raising cell potential to undesirably high values. Overcharge may cause electrolyte breakdown, electrode degradation, current collector corrosion, gas evolution and so on [1]. Meanwhile, increased internal pressure and generated heat increase the risk of explosions and fires.

The common external methods for overcharge protection add weight, cost, and complexity to the battery pack. Redox shuttles, as a reversible internal protection mechanism, are widely used to allow for continued operation in the event of overcharge [2-4]. However, the overcharge performance is fundamentally limited due to redox shuttle solubility, diffusion coefficient, and chemical stability [5]. In recent years, an alternative approach using electroactive polymers for overcharge protection has aroused the attention of researchers. When incorporated into a battery separator, electroactive polymers can provide a reversible internal short upon overcharging to maintain the cell potential within a safe range. According to physical properties of electroactive polymers, various film-forming methods have been discussed, such as roll pressing [6], impregnating [7], electrospinning [8] and so on. The film-forming method seriously affects utilization and stability of the polymers. Therefore, the film-forming methods of electroactive polymers hold considerable future promise.

In this study, we tried to synthesize an inexpensive electroactive polymer polytriphenylamine (PTPAn), which can be oxidized to a conductive state at a higher potential. The composite separator was prepared simply by impregnating a commercial porous separator with PTPAn polymer solution. The microstructure and electrochemical property of the composite separator were evaluated. The overcharge property of blocking cells assembled with the composite separator and the commercial PP separator was also investigated..

\section{Experimental}

\subsection{Preparation of polytriphenylamine}

The triphenylamine (TPAn) of $0.025 \mathrm{~mol}$ was dissolved in $100 \mathrm{~mL}$ chloroform to form a mixed solution, with brief ultrasonic treatment to speed dissolution. Then 0.1 mol $\mathrm{FeCl}_{3}$ oxidant (4 times than TPAn) was added into the reaction solution within an hour. The oxidative polymerization of TPA usually continued for $12 \mathrm{~h}$ in an inert gas condition. After that, the reaction mixture was poured into methanol to deposit the polymer product, which was then filtered and washed with methanol several times. In order to purify the coarse PTPAn, the collected polymer powders were dissolved again in chloroform to remove the insoluble residues by filtration. The filtrate was re-deposited with methanol. Finally, the polymer product was filtered and dried in vacuum at $60{ }^{\circ} \mathrm{C}$ for $24 \mathrm{~h}$.

\subsection{Preparation of the composite separator}

The PTPAn-modified separator was prepared using a dipcoating process. Firstly, the electroactive polymer of PTPAn was dissolved in chloroform to form a $2 \mathrm{wt.} \%$ polymer solution. Then the PTPAn-modified separator was prepared simply by immersing a commercial PP separator $($ Celgard 2500 , thick $=25 \mu \mathrm{m}$, porosity $=55 \%$ ) into the polymer solution for $10 \mathrm{~min}$ under ultrasonic

\footnotetext{
a Corresponding author: wxiao@imr.ac.cn
} 
condition, evaporating the solvents off to leave a modified separator. The resulting composite separator was $27 \mu \mathrm{m}$ thick and the mass gain is about $0.2 \mathrm{mg} / \mathrm{cm}^{2}$ after the PTPAn coating progress.

\subsection{Characterization of the composite separator}

The surface morphology of the separators was examined using a scanning electron microscopy (JEOL, JSM-6300). The phase structure of prepared PTPAn was studied using FT-IR (IRTracer-100) and XRD (D/max2200VPC). The thermal shrinkage of the separators was determined by measuring the dimensional change before and after being exposed at $140{ }^{\circ} \mathrm{C}$ for $0.5 \mathrm{~h}$. The electrochemical behavior of prepared PTPAn was examined by cyclic voltammetry (CV). The working electrode used for $\mathrm{CV}$ was a PTPAn-coated Al foil electrode, which was prepared by impregnating Al foil with a chloroform solution of PTPAn, and then taking out for evaporating the solvents off to leave a PTPAn coating layer on the $\mathrm{Al}$ foil electrode. The $\mathrm{CV}$ curves were recorded on an electrochemical workstation (Princeton Applied Research 273, USA) using a two-electrode CR2032-type cell with PTPAn-coated Al foil electrode as working electrode and a $\mathrm{Li}$ foil as both counter electrode and reference electrode in the electrolyte $\left(1.0 \mathrm{M} \mathrm{LiPF}_{6}\right.$ in $\mathrm{EC} / \mathrm{DEC}$ ). The overcharge performance of the composite separator and the commercial PP separator was evaluated in a blocking cell with the composite separator sandwiched between a stainless steel current collector and a $\mathrm{Li}$ foil in the electrolyte. After a $30 \mathrm{~min}$ rest period, increasing currents were applied to the cell, each holding for $10 \mathrm{~min}$ before stepping to the next current level and the cell potential was recorded.

\section{Results and Discussion}

It is well known that PTPAn can be synthesized by chemical and electrochemical polymerization of TPA. In contrast, PTPAn produced from chemical polymerization has a higher degree of polymerization and structural homogeneousness. Therefore, we adopted chemical polymerization to prepare PTPAn.

The FTIR spectra of TPAn and PTPAn are shown in Fig. 1. The main vibration bands characteristic of TPAn can also be found in PTPAn, such as C-C stretching at 1491 $\mathrm{cm}^{-1}, \mathrm{C}-\mathrm{H}$ bending at $1322 \mathrm{~cm}^{-1}$ and $\mathrm{C}-\mathrm{N}$ stretching at $1274 \mathrm{~cm}^{-1}$. Compared with TPAn, a new band at $819 \mathrm{~cm}^{-1}$ appears in PTPAn, which belongs to the $\mathrm{C}-\mathrm{C}$ bending between the benzene rings of TPA. In addition, the peak at $1275 \mathrm{~cm}^{-1}$ is broader than that in the TPAn and the peak at $1596 \mathrm{~cm}^{-1}$ is shifted from its position in the monomer to higher wave numbers. In the region 900 to $1200 \mathrm{~cm}^{-1}$, the peak profile has changed due to higher concentration of para substituted phenylene rings in PTPAn. These results suggest a highly crosslinked structure of the PTPAn polymer, which is consistent with previous study $[9,10]$.

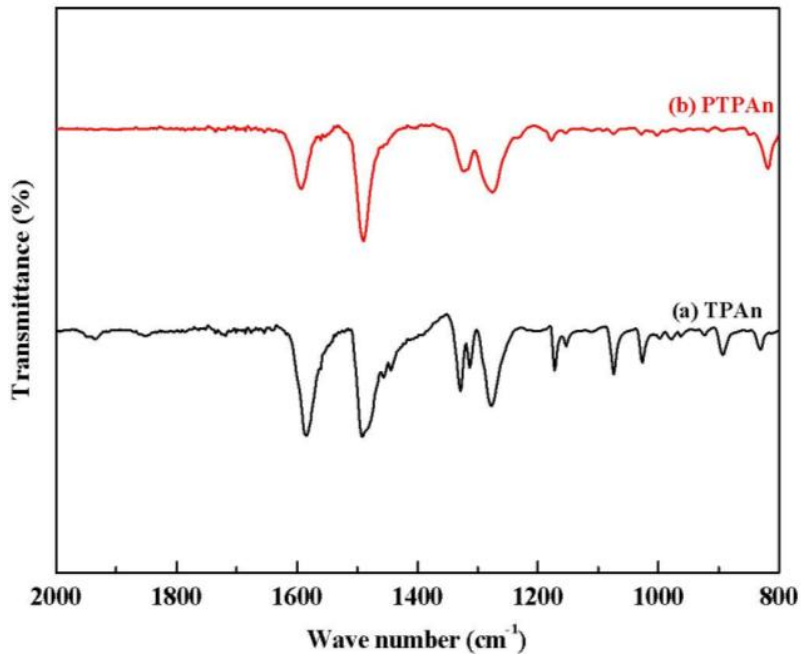

Figure 1. The FTIR spectra of TPAn and PTPAn.

Fig. 2 illustrates the XRD patterns of TPAn and PTPAn. It is obvious that TPAn exists as a crystal phase. After oxidation polymerization, the crystallographic structure has been significantly changed. And a single broad diffraction peak recorded at $20^{\circ}$ can be found, indicating that TPAn can entirely change into PTPAn (amorphous phase).

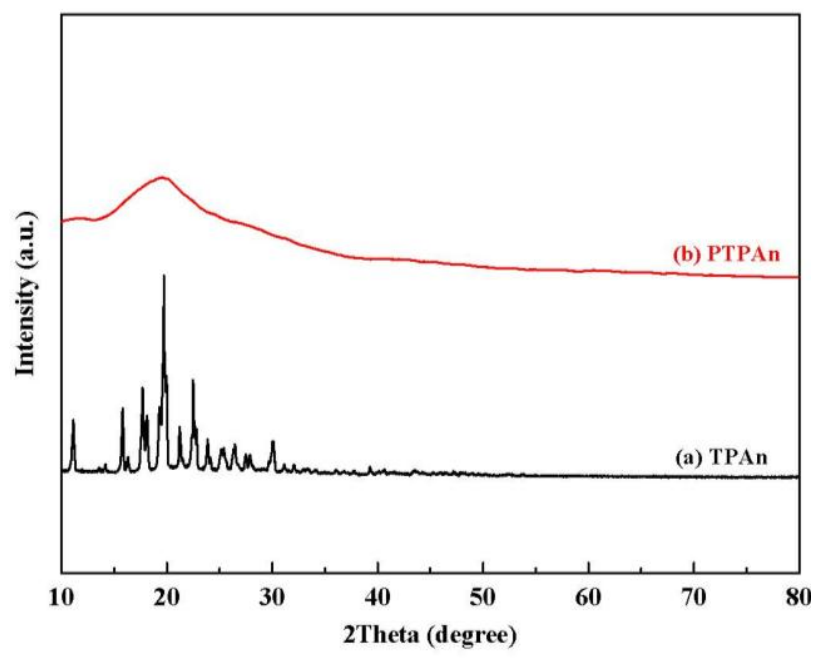

Figure 2. The XRD patterns of TPAn and PTPAn.

Fig. 3 shows the CV curves of PTPAn.The PTPAn polymer exhibits a pair of overlapped redox peaks between 3.0 and $4.5 \mathrm{~V}$, which can be regarded as a reversible electrochemical p-doping and dedoping process. The anodic and cathodic branches of PTPAn are very symmetric in the peak height and peak areas, which contributes to reversible and rapid electrochemical processes of the polymer. In the doping process (forward scan), the anodic current of PTPAn appears at the onset potential of $3.7 \mathrm{~V}$. In the dedoping process (reverse scan), the cathodic current of PTPAn starts at the reversed potential of $3.8 \mathrm{~V}$ and reaches its maximum current at 3.5 $\mathrm{V}$. These results indicate that the PTPAn can be used to provide reversible overcharge protection. 


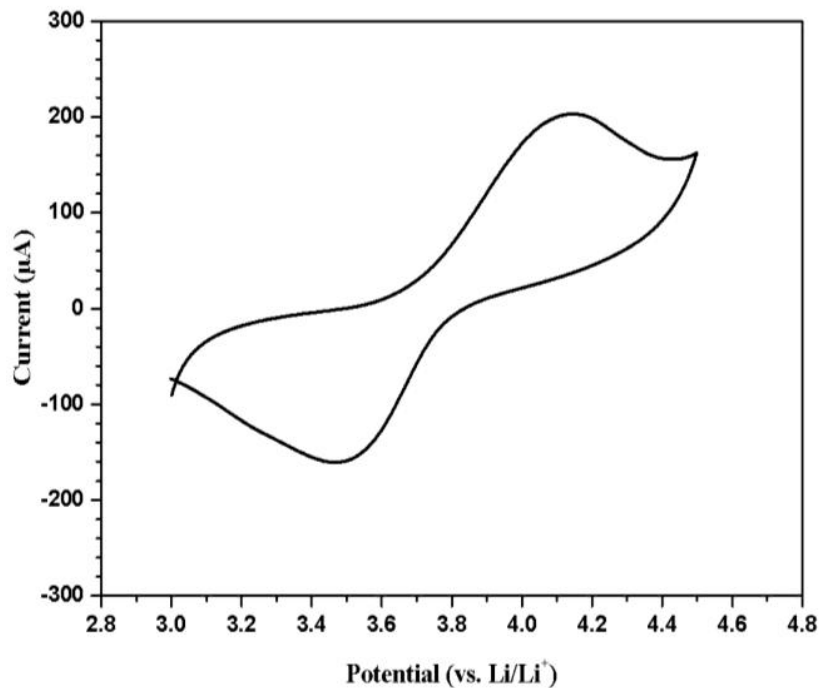

Figure 3. The CV curve of PTPAn. Scan rate: $10 \mathrm{mVs}^{-1}$.

The surface morphologies of the PP separator and the composite separator are shown in Fig. 4. The PP separator exhibits a uniform porous structure. After modification, PTPAn is observed on the surface of the composite separator and forms a uniform coating. Also PTPAn polymer exists within the PP separator pores and does not completely block the pores.

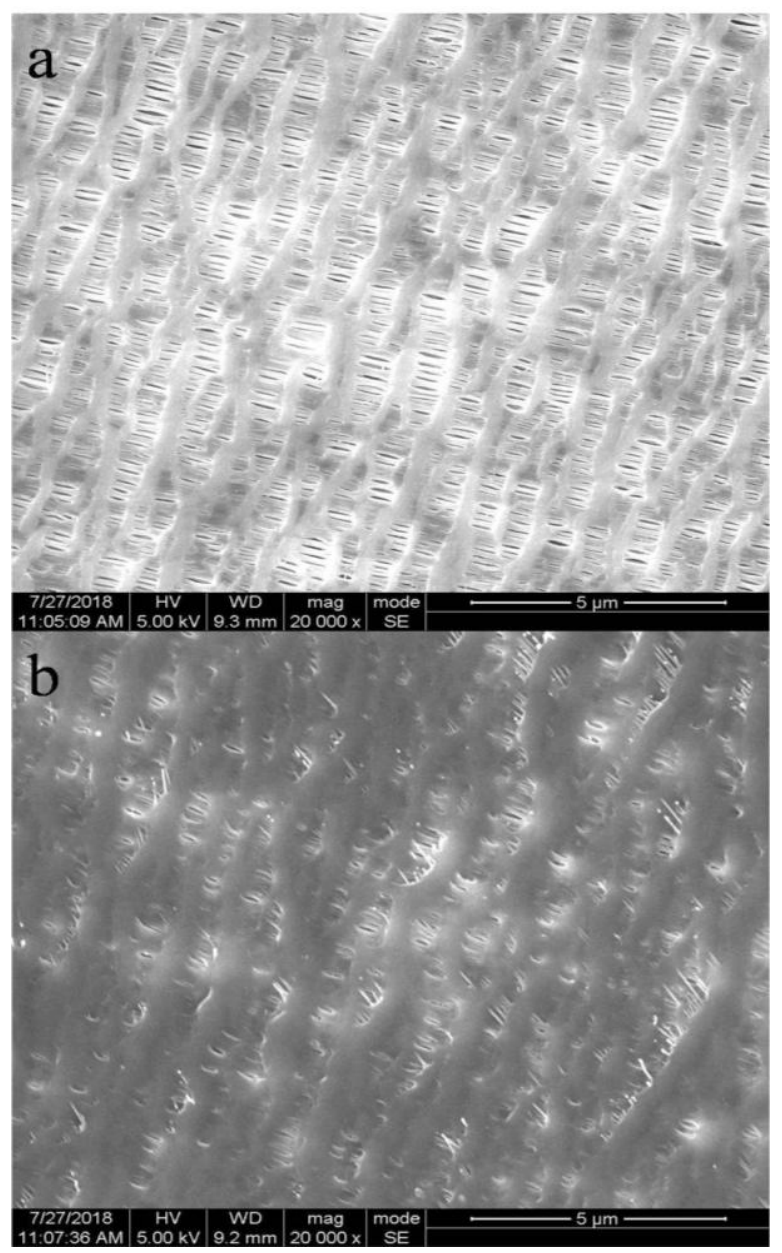

Figure 4. The surface morphologies of (a) the PP separator and (b) the composite separator
Fig. 5 shows the photographs of the PP separator and composite separator before/after being exposed to $140{ }^{\circ} \mathrm{C}$ for $0.5 \mathrm{~h}$. Since the PP separator has a melting point of about $160{ }^{\circ} \mathrm{C}$, it easily loses dimensional stability upon $140{ }^{\circ} \mathrm{C}$. While the composite separator distinctly suppress the thermal shrinkage compared with the PP separator owing to the existence of the electroactive polymer PTPAn. As with other polymer materials, the heat resistant PTPAn is believed to effectively prevent the composite separator from being thermally shrunk.

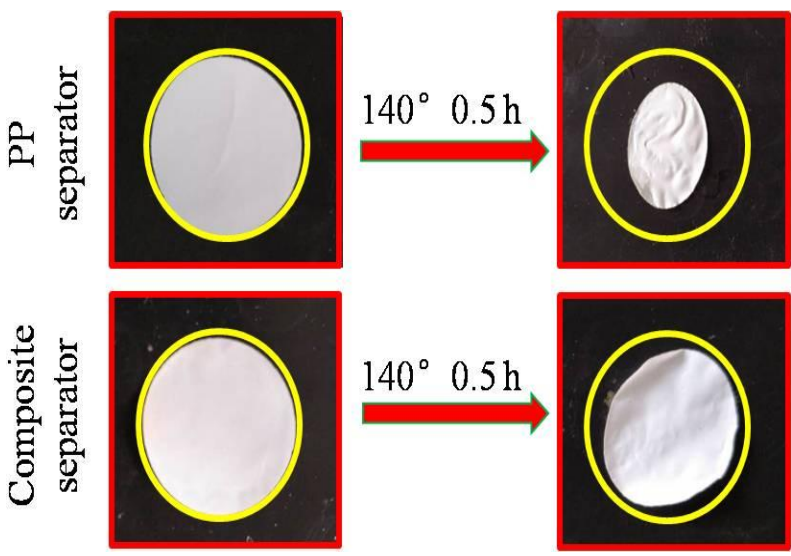

Figure 5. The photographs of the PP separator and composite separator before/after being exposed to $140{ }^{\circ} \mathrm{C}$ for $0.5 \mathrm{~h}$.

Fig. 6 shows the voltage profile of the blocking cells with the PP separator and composite separator at various applied current densities. As shown in Fig. 6, the cell with PP separator can not remain on a constant potential. And the potential increases sharply with the increase of applied current density. While the cell with the composite separator is able to hold a constant potential for current densities up to $5 \mathrm{~mA} / \mathrm{cm}^{2}$, which is ascribed as the electrochemical p-doping process of PTPAn. The $p$ doping process results in cell short. Therefore, the composite separator can maintain the cell potential within a safe range in case of overcharging based on its reversible internal short.

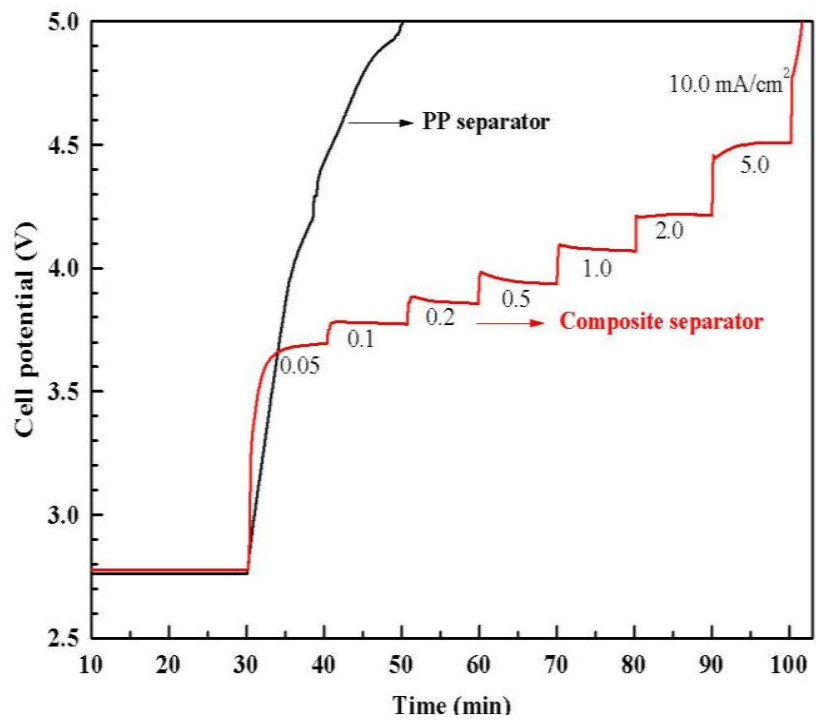

Figure 6. The voltage profiles of the blocking cells with the PP separator and composite separator at various current densities. 


\section{Conclusion}

The composite separator was successfully prepared by impregnating a commercial PP separator with PTPAn polymer solution. PTPAn was observed on the surface of the composite separator and formed a uniform coating. The PTPAn polymer exhibited a pair of well-defined redox peaks between 3.0 and $4.5 \mathrm{~V}$, which could be regarded as a reversible electrochemical p-doping and dedoping process. When incorporated in a blocking cell, the composite separator was able to hold a constant potential for current densities up to $5 \mathrm{~mA} / \mathrm{cm}^{2}$ to protect the cell from overcharging.

\section{Acknowledgements}

This work was supported by the National Natural Science Foundation of China (No. 21676282), Natural Science Foundation of Liaoning (No. 20180510039) and Shenyang Science and Technology Project (No. 18013051).

\section{References}

1. S. A. Odom, S. Ergun, P. P. Poudel, R. P. Sean, Energy Environ. Sci. 7, 760 (2014)

2. Z. Zhang, L. Hu, H. Wu, W. Weng, M. Koh, P. C. Redfern, L.A. Curtissb, K. Aminead, Energy Environ. Sci. 6, 1806 (2013)

3. W. Weng, Z. Zhang, P.C. Redfern, L.A. Curtiss, K. Amine, J. Power Sources 196, 1530 (2011)

4. B. Wang, T. J. Richardson, G. Chen, Phys. Chem. Chem.Phys. 15, 6849 (2013)

5. L. Zhang, Z. Zhang, H. Wu, K. Amine, Energy Environ. Sci. 4, 2858 (2011)

6. X.M. Feng, J.Y. Zheng, J.J. Zhang, R.F. Li, Z.J. Li, Electrochim. Acta 54, 4036 (2009)

7. S.L. Li, L. Xia, H.Y. Zhang, X.P. Ai, H.X. Yang, Y.L. Cao, J. Power Sources 196, 7021 (2011)

8. B. Wang, T.J. Richardson, G. Chen, J. Electrochem. Soc. 161, A1039 (2014)

9. J.K. Feng, Y.L. Cao, X.P. Ai, H.X. Yang, J. Power Sources 177, 199 (2008)

10. A. Petr, C. Kvarnstrom, L. Dunsch, A. Ivaska, Synthetic Met. 108, 245 (2000) 